\title{
Quality of Life in Patients with Alzheimer's Disease as Reported by Patient Proxies.
}

\author{
S.M. Albert, PhD, ' C. Del Castillo-Castaneda, MA," M. Sano, PhD, 'D.M. Jacobs, PhD, \\ K. Marder, MD, ${ }^{*}$ K. Bell, MD, ${ }^{*}$ F. Bylsma, PhD, ${ }^{\dagger}$ G. Lafleche, PhD, ${ }^{\ddagger} J$. Brandt, PhD, ${ }^{\dagger}$ \\ M. Albert, PhD, and Y. Stern, PhD
}

OBJECTIVE: To measure behaviors indicative of quality of life (QOL) in patients with Alzheimer's disease and to examine correlates of patient QOL.

DESIGN: Cross-sectional investigation.

SETTING: Multi-center study.

PARTICIPANTS: Sample of 130 diagnosed patients.

PRINCIPAL OUTCOME MEASURES: Proxy ratings of (1) the frequency, opportunity, and enjoyment of 15 non-ADL activities potentially within the capacity of a demented person, and (2) the frequency of a series of positive and negative affects, evident in clearly demarcated facial and bodily expressions.

RESULTS: QOL ratings were reliably elicited. Family and institutional caregivers differed only in reports of opportunity for patient activity. Frequency of activities declined with increasing severity of dementia. The frequency of negative affects increased and positive affects declined with increasing severity of dementia, but correlations were weak. High QOL, defined by frequent activity and positive affect, was evident in a quarter of the sample. In multivariate models, functional and cognitive status independently predicted QOL among community-resident older adults; only absence of antipsychotics was related to QOL among older people in nursing homes. Patient education, a marker of premorbid state, independently predicted some activity patterns.

CONCLUSIONS: Although the subjective world of the demented patient is not directly accessible, readily observable behaviors offer a basis for assessing QOL. J Am Geriatr Soc 44:1342-1347, 1996.

From the "Gertrude H. Sergievsky Center and Department of Neurology, College of Physicians and Surgeons, Columbia University, New York; tDepartment of I'sychiatry and Behavioral Sciences, Johns Hopkins University, Baltimore, Mary land; and Departments of Psychiatry and Neurology, Harvard Medical School, Boston, Massachusetts.

Address correspondence to S.M. Albert, PhD, Gertrude H. Sergievsky Center, Columbia University, $630 \mathrm{~W} 168 \mathrm{th}$ St., New York, NY 10032.
$\mathrm{D}$ ementia involves progressive cognitive and functional decline, which, in the late stages of disease, finally makes it impossible for the demented person to experience the world in many of the ways we take for granted in daily life. ${ }^{1}$ For the ethicist, severe cognitive deficit jeopardizes essential features of personhood, such as the capacity to "envisage and desire a future for oneself." 2 For the family caregiver, severe cognitive deficit means irrevocable change in a spouse or a parent, often phrased as a "loss" of the person.

Is it sensible, then, to speak of the quality of life (QOL) of someone in such a state? One response is simply to deny the relevance of quality of life inquiry; in this view, demented people do not experience the world in any meaningful sense. But this view is surely too strong, for dementia is not a single, psychologically null state (as in this case of severe coma), but admits gradations, with variable impact on activity and experience.

An alternative view is to admit that whereas dementia patients do not experience the world as we do, many do have an "experiential world," and we know very little about it." We do not know what dementia patients experience because they cannot tell us, but also because we have not attempted to measure features of their daily lives that may convey information about this experience. Even if the subjective world of the demented patient is not directly accessible, readily observable behaviors may offer insight into that world and, hence, a basis for assessing patient QOL. In fact, little research is available, though this has not stopped bioethicists from assuming, for example, that "the severely demented ... approach more closely the condition of animals than normal adult humans in their psychological capacities." 2

\section{For editorial comment, see p 1400}

We have used this measurement approach to design an instrument for assessing QOL in dementia. Here we describe the development and reliability of the instrument. We also present a cross-sectional analysis of predictors of QOL in a cohort of diagnosed Alzheimer's patients. We suggest that QOL in Alzheimer's disease can be rated reliably and offers insight into the experience of dementia.

An additional significance of the QOL measure, which will become more significant as follow-up of the cohort continues, is worth noting. It is clear that many measures of disease course, such as the Blessed Dementia Rating Scale ${ }^{4}$ and the modified Mini-Mental State Examination, ${ }^{5,6}$ suffer from "floor effects"; at severe stages of disease, the measures 
are unable to discriminate among patients who may still show variation in preserved capacities. Because the proposed QOL measure is based on extremely simple measures of activity and affect, it may be useful as an additional marker of disease course, showing variablity among patients who have already "bottomed out" on standard measures of cognitive and functional ability. In fact, preliminary data suggest that the measure has this desirable property, which we present here as well.

\section{METHODS}

\section{Measures}

The quality of life measures used in this research involve two domains, patient activity and affect, and represent adaptations of existing measures. These domains were chosen because (1) each can be assessed in terms of behaviorally anchored, countable events and are likely to be rated reliably as a result, and (2) the two are also likely to be related to the unobservable, subjective experience of patients. That is, we assumed that demented patients who participate in a greater number of activities within their capacity and who appear to be content or interested in what is going on around them are likely to have a different quality of experience, other things being equal, than demented patients who do not show this level of engagement.

For our measure of activity, we modified Teri and Logsdon's "Pleasant Events Schedule-AD," a set of activities judged to be within the capacity of demented individuals receiving supervision and aid in daily activities. ${ }^{7}$ We reduced the original 53-item scale to a set of 15 items that excluded personal and instrumental care activities. These activities were chosen to be appropriate for both urban and non-urban, and community and nursing home, populations; the reduced set was also necessary for telephone administration. For each activity, we obtained a frequency count, an opportunity count, and a current enjoyment count. We altered the scaling of the items so that they were suitable for a 1-week time frame, limiting responses to "frequently" ( $\geq 3 \times /$ week), "occasionally" $(<3 \times / \mathrm{wk})$, or "never" for the activity and opportunity measures. Current enjoyment was coded dichotomously. The activities assessed in this measure cover a wide range of behaviors, including going outside, going for a ride in a car, visiting with family and friends, exercising, reading or being read a story, going to a museum or watching a movie, and working on a craft. We also asked proxies to report on whether patients engaged in a task they thought "difficult" or challenging to the patient. A summary activity measure was defined as the sum of the frequency in which patients performed the set of 15 activities during the previous week (range $0-30$ ).

Norms for the frequency of such activities among demented older people are lacking. As a result, we inspected the distribution of activity scores for the sample and defined "high" activity as a score greater than the median of this distribution.

For the measure of affect, we used Lawton's "apparent emotion" items, a set of six affects, both positive and negative, that have been judged to be recognized commonly and described easily in terms of facial and body expression. ${ }^{8}$ The measure is similar to the "Discomfort Scale" proposed for Alzheimer's patients. ${ }^{9}$ Both rely on behavioral indicators of emotional states and assess positive as well as negative affects. The affects include pleasure, anger, anxiety, depression, in- terest, and contentment. Each affect is specified with a clear set of physical descriptors and coded in terms of frequency during the previous 2 weeks. For example, "anger" is described in terms of "clench teeth, grimace, shout, curse, berate, push, or physical aggression"; pleasure is described in terms of "smile, laugh, touching, singing, or open-arm gestures." The frequency of affect was coded in a 5-point format, ranging from never to $\geq 3 \times /$ day. Summary positive (sum of frequency of happy, interested, and content; range 3-15) and negative (sum of frequency of angry, depressed, and anxious; range 3-15) affect scores were computed because the affects formed well-defined composites in factor analyses.

For the affect measures, norms are again unavailable for community-dwelling demented older adults. We relied on an a priori definition of "high positive" and "low negative" affect. Subjects who were reported to experience all three of the positive affects at least $3 \times /$ day (the maximum score) were considered to have high positive affect. Subjects who were reported to express all three of the negative affects less than $1 \times /$ day were considered to have low negative affect. The activity and affect indicators were combined to construct a composite QOL indicator. High QOL was defined in terms of high positive affect and high activity, reflecting the requirement that quality of life gives equal weight to both a subjective indicator (affect) and a more objective indicator (activity). ${ }^{10}$

Other measures included patients' dementia status as reflected in functional ability, ${ }^{4}$ cognitive status, ${ }^{5,6}$ depression, ${ }^{11}$ and psychiatric symptoms. ${ }^{12}$ For cognitive status, the 57-item modified Mini-Mental State Exam (mMMS) was used; this assessment supplements the original Folstein MiniMental State Exam with the Wechsler Digit Span test and additional tests of attention, language, and calculation. ${ }^{6}$ The range of $\mathrm{mMMS}$ scores was divided into four categories: a score of $0,1-20,21-40$, and $>40$. Neurologists also staged each patient according to the Clinical Dementia Rating scale $^{13}$ and examined patients for the presence of extrapyramidal signs. Finally, sociodemographic data and information on comorbid illnesses were collected.

\section{Procedures}

Assessment of quality of life measures began during the sixth to eighth follow-up evaluation of subjects enrolled in the "Predictors Cohort," a multi-site longitudinal study of patients with Alzheimer's disease. Beginning in 1988, patients were recruited into the study from Columbia University, Johns Hopkins University, and Massachusetts General Hospital. Patients were drawn from memory disorders clinics, neurology practices, and participants in drug trials. Patients in this cohort receive detailed evaluations every 6 months. To be included in the cohort, patients had to meet DSM-III criteria for dementia along with NINDS-ADRDA criteria for probable AD. In addition, subjects recruited were at a relatively mild stage of dementia, which was defined as a mMMS score $\geq 30$. $^{14,15}$

Trained interviewers collected the affect and activity information in a telephone interview conducted with caregivers. Telephone contact with patient proxies for the QOL assessment occurred within 2 weeks of patient examinations.

The original cohort consisted of 236 patients. At the sixth follow-up interval, when QOL assessment began, 155 patients were still alive and actively followed. Of the 155 patients, 130 patients $(83.9 \%)$ have received at least one assessment with both the affect and activity measures; five of 
these patients provided incomplete information, requiring them to be excluded from some of the analyses presented below. These data continue to be collected in subsequent assessments; here we report on cross-sectional findings only.

\section{Statistical Methods}

Univariate tests of differences in proportions were conducted with reference to $t$ (continuous variables) or $\chi^{2}$ (categorical variables) distributions, as appropriate. Pearson correlations were computed between individual QOL indicators and $m M M S$ scores to assess the effect of dementia severity on QOL. Multivariate analyses involved logistic regression models to assess the effect of cognitive and functional deficit on QOL, independent of age, gender, and education. Regression models for continuous variables were also developed to assess the relation between cognitive performance and components of QOL (affect and activity). In these models, a quadratic term was introduced to assess curvilinear relationships. Regression models were also developed to assess the relation between premorbid educational level and activities in dementia. In these models, cognitve performance was entered as a covariate to see if education was related to activities controlling for severity of dementia.

To assess test-retest reliability of the QOL measures, the measures were fielded twice, with a subsample of 10 patients, 1 week apart. For the continuous affect variables, correlations were computed as a measure of agreement. For the categorical activity measures, we followed Fleiss ${ }^{16}$ and computed kappa. Because it corrects for chance agreement, kappa is a stringent test of reliability. Values above .60 are considered excellent and above .40 , acceptable.

\section{RESULTS}

Table 1 shows features of the cohort, comparing baseline characteristics of those receiving the QOL assessment and those who did not receive the assessment. The sample was largely white (5\% non-white). As shown in the table, patients entered the study at about age 73 , were more likely to be women, and were highly educated. Most were living in the community, but patients not living in the community at baseline were significantly less likely to receive the QOL assessment in the course of the study. Most patients had mild dementia at study entry, as indicated by Clinical Dementia Rating staging, and mid-range impairment on measures of cognition and function. A small proportion of patients had comorbid conditions. Aside from living arrangement, patients receiving the QOL assessment did not differ from other patients in the cohort.

At the time of the QOL assessment (6th-8th follow-up visit), $65.6 \%(82 / 125)$ of patients were still living at home. More than half the sample (59\%) had severe ADL limitations (required help or could not do two of three basic ADL tasks: dressing, grooming, or toileting). Also, more than half the sample $(51 \%)$ had symptoms of psychiatric disorder (hallucinations, delusions, or agitation). More than one-quarter of the sample $(28.7 \%$ ) had floor values (score of 0 ) on the mMMS, and $33.9 \%$ of subjects had severe or profound AD by CDR staging. About one-third of the subjects were currently taking either an antidepressant, antipsychotic, or sedative. The distribution of mMMS scores included 13 subjects with a score of 0,33 in the 1 to 20 range, 46 in the 21 to 40 range, and nine subjects with scores greater than 40; no subject had a score higher than 53 .
Table 1. Baseline Comparison of AD Patients Receiving Quality of Life Assessment and Patients Not Receiving Assessment

\begin{tabular}{|c|c|c|c|}
\hline & $\begin{array}{l}\text { Subjects } \\
\text { Assessed } \\
\text { for QOL } \\
(n=130)\end{array}$ & $\begin{array}{l}\text { Subjects } \\
\text { Not Assessed } \\
\text { for QOL } \\
(n=106)\end{array}$ & $P$ \\
\hline \multicolumn{4}{|l|}{ Sociodemographics } \\
\hline Age at Intake (yrs) & $72.4( \pm 8.1)$ & $73.9( \pm 9.7)$ & .226 \\
\hline Gender ( $\%$ female) & 60.5 & 57.9 & .696 \\
\hline Education (yrs school) & $13.2( \pm 3.6)$ & $13.0( \pm 3.6)$ & .736 \\
\hline $\begin{array}{l}\text { Living in community } \\
(\%)\end{array}$ & 95.3 & 85.9 & .036 \\
\hline \multicolumn{4}{|l|}{ Function } \\
\hline $\begin{array}{l}\text { BDRS (total score) } \\
\text { mMMS (total score) }\end{array}$ & $\begin{array}{l}7.96( \pm 3.5) \\
37.8( \pm 5.9)\end{array}$ & $\begin{array}{l}8.07( \pm 3.5) \\
38.0( \pm 5.2)\end{array}$ & $\begin{array}{l}.803 \\
.730\end{array}$ \\
\hline \multicolumn{4}{|c|}{$\begin{array}{l}\text { Clinical Dementia Rating } \\
\text { Scale }\end{array}$} \\
\hline Questionable (\%) & 3.1 & 4.7 & \\
\hline Mild (\%) & 83.7 & 89.7 & \\
\hline Moderate (\%) & 13.2 & 5.6 & $.131^{*}$ \\
\hline \multicolumn{4}{|l|}{ Comorbidities } \\
\hline Past MI (\%) & 4.8 & 6.5 & .567 \\
\hline Angina $(\%)$ & 8.6 & 11.2 & .515 \\
\hline $\mathrm{CHF}(\%)$ & 2.4 & 4.7 & .342 \\
\hline Malignancy (\%) & 1.6 & 5.6 & .108 \\
\hline Diabetes (\%) & 4.8 & 6.5 & .567 \\
\hline
\end{tabular}

BDRS = Blessed Dementia Rating Scale; $m$ MMS = Modified Mini-Mental State. $\chi^{2}$ test for significance; all other significance tests based on $t$ ratio.

\section{Reliability}

The affect and activity measures were received readily by caregivers and posed no difficulty in administration. In the 1-week test-retest assessment of measures, correlations between the two administrations of the 5-point affect items were on the whole adequate (depression, .80; anxiety, .70; anger, .92; contentment, .87; interest, .74; pleasure, .53). Kappa was above .40 for 13 of the 15 activity items and above .60 for 12 of the 15 items. The two items with low kappa values were "entertainment" (.14) and "radio/TV" (.34). Reliability for the dichotomous enjoyment questions was excellent (for 14 of 15 items, kappa $>.70$ ). Only "radio/ TV" had a low kappa for enjoyment $(.25)$.

\section{Validity}

Strictly speaking, validity of the measures cannot be assessed without a true gold standard, in this case observations of patient behavior by a clinician rater. In this predominantly community-based sample, such a gold standard is not available. To assess validity, therefore, we relied on more indirect measures. First, we assessed the responses of family caregivers relative to institututional caregivers. If the two caregiver types rate patients similarly, it is less likely that family caregivers are giving biased reports of patient behavior. Second, we assessed QOL relative to measures of dementia severity. Patients with more severe disease should have lower QOL. For this last comparison, performance on the mMMS was used as an indicator of severity of dementia.

\section{Family-and Institutional-Caregiver Ratings of $Q O L$}

Table 2 compares the QOL ratings of family $(n=118)$ and institutional ( $n=12$ ) caregivers. The upper panel of the table 
Table 2. Comparison of Family and Institutional Caregiver Reports on Paticnt QOL

\begin{tabular}{lccc}
\hline & $\begin{array}{c}\text { Family } \\
\text { Caregivers }\end{array}$ & $\begin{array}{c}\text { Institutional } \\
\text { Caregivers }\end{array}$ & $P$ \\
\hline All patients & & & \\
Activity & & & \\
$\quad$ Opportunity $(\mu)$ & $14.5( \pm 5.1)$ & $6.8( \pm 1.9)$ & $<.001$ \\
$\quad$ Frequency $(\mu)$ & $11.2( \pm 5.3)$ & $5.3( \pm 2.8)$ & $<.001$ \\
$\quad$ Enjoyment $(\mu)$ & $6.6( \pm 3.4)$ & $4.1( \pm 2.5)$ & .013 \\
Emotion & & & \\
$\quad$ High positive (\%) & 34.8 & 25.0 & .499 \\
$\quad$ Low negative (\%) & 55.2 & 41.7 & .376 \\
Patients in nursing & & & \\
$\quad$ homes & & & \\
Activity & & $6.8( \pm 1.9)$ & $<.001$ \\
$\quad$ Opportunity $(\mu)$ & $12.3( \pm 5.4)$ & .215 \\
$\quad$ Frequency $(\mu)$ & $7.3( \pm 5.3)$ & $5.3( \pm 2.8)$ & .905 \\
$\quad$ Enjoyment $(\mu)$ & $4.2( \pm 3.6)$ & $4.1( \pm 2.5)$ & .901 \\
Emotion & & & .522 \\
$\quad$ High positive (\%) & 35.5 & 25.0 & .764 \\
$\quad$ Low negative (\%) & 46.9 & 41.7 & \\
\hline
\end{tabular}

All patients: 118 family and 12 institutional caregivers providing QO1. reports. patients in nursing homes: 32 family and 12 institutional caregivers.

Activity: Opportunity and frequency (mean of 15 activities, range 0-30); enjoyment, mean of 15 activities enjoyed (range $0-15$ ). High positive: $>3 \times /$ day on all three positive affects (happy, content, interested). Low negative: $<1 \times /$ day on all three negative affects (anger, anxiety, depression).

shows the overall superior QOL of patients receiving care from family caregivers. These caregivers report greater opportunity, frequency, and enjoyment of the 15 patient activities assessed, but no differences in levels of positive or negative affect. A more stringent comparison is presented in the lower panel, which compares family and institutional caregiver ratings among patients in nursing homes. Of the 43 patients in institutional care, 32 family members and 12 institutional caregivers served as patient proxies for the QOL assessment. Comparing the two sets of reports shows that family caregivers do not differ from institutional caregivers except in the case of opportunity for activity, a plausible difference representing family members' additional efforts in attending to patients. Thus, family and institutional caregivers report on patient $\mathrm{QOL}$ in similar ways, and family members' reports do not appear to be biased according to this standard.

\section{QOL and Severity of Dementia}

More severe dementia should be associated with poorer QOL, that is, reduction in activity, and perhaps lower positive affect and greater negative affect. To assess these relationships, we plotted the summary indicators of QOL against scores on the mMMS and also examined correlations between $\mathrm{mMMS}$ scores and each indicator of QOL.

Figure 1 shows that cognitive status and activity are inversely correlated. The lower the mMMS score, the less opportunity, frequency, and enjoyment of the 15 activities. The correlation is significant $(P<.001)$ for all indicators.

Figure 2 shows the frequency of five representative activities and indicates considerable variability in activities across stage of cognitive decline. Visual inspection shows that some activities appear to decline with increasing severity of dementia (Radio/TV, Go outside, Reading); some appear fairly flat

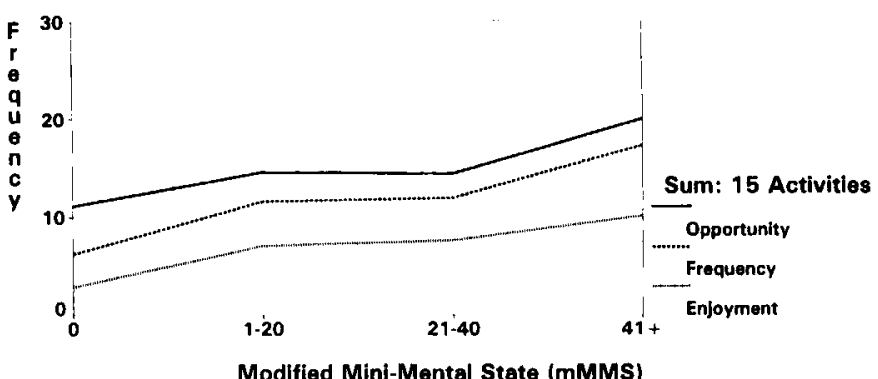

Figure 1. Cognitive deficit in $\mathrm{AD}$ and summary indicators of caregiver-supported activity. Opportunity and frequency: Sun of 15 activities (range $0-30$ ). Enjoyment: Number of activities currently enjoyed.

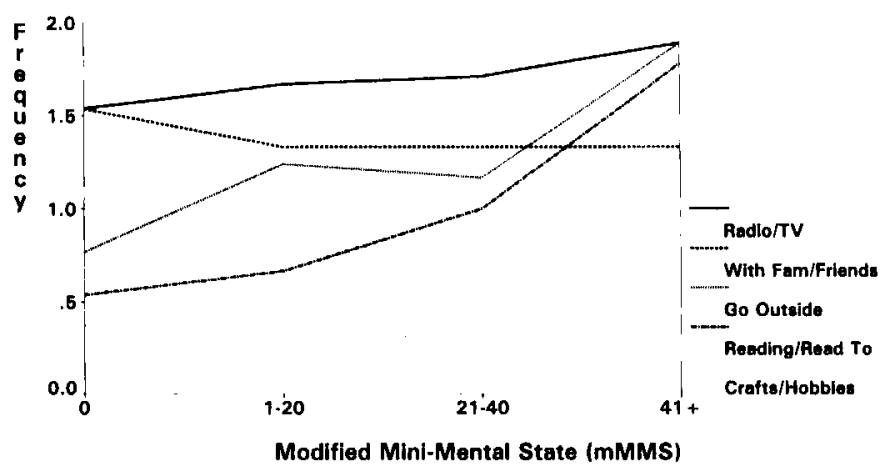

Figure 2. Cognitive deficit in $\mathrm{AD}$ and activity. Mean frequency: 0 , never; $1,<3 \times / \mathrm{wk} ; 2,>3 \times / \mathrm{wk}$.

across the spectrum of cognitive ability (Crafts/hobbies), declining only at the most severe levels of dementia; and some appear to increase with more severe dementia (time with family/friends), showing the effect of increasing caregiver involvement. A significant correlation between activity and severity of dementia is evident for reading behavior $(r=.376$, $P<.001)$; other correlations were not significant.

Visual inspection of the frequency of negative affects, shown in Figure 3, shows an apparent increase in the negative affects with increasing severity of dementia (anxiety, $r=$ $-.129[P=.193]$; anger, $r=-.165[P=.100]$; depression, $r=-.152(P=.128])$; but these correlations do not achieve statistical significance Inspection of the figure shows that anxiety is highest at intermediate stages of cognitive decline. This curvilinear relationship was assessed by regressing anxiety on mMMS and the square of mMMS. While the quadratic term was not significant for the sample as a whole, it

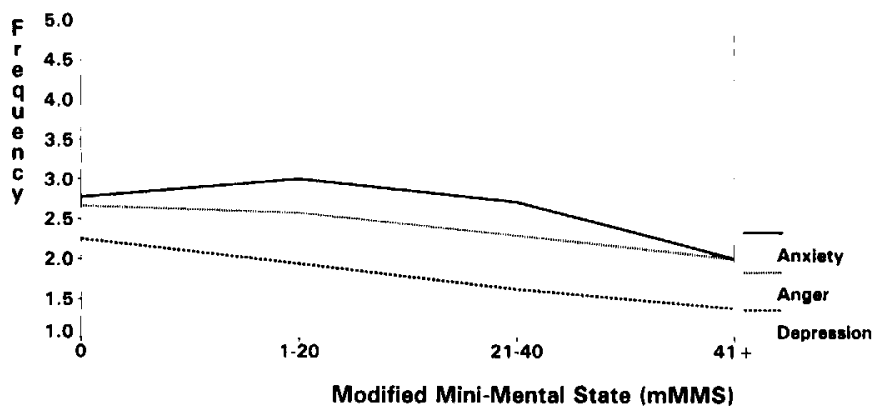

Figure 3. Cognitive deficit in AD: Effect on frequency of negative affect. Mean frequency: 1 , never; $5,>3 \times /$ day. 
was in the subsample of patients using antipsychotic medications $(P=.011)$.

The pattern for the positive affects is shown in Figure 4. Interest in the world was significantly less frequent among subjects with more advanced dementia $(r=.377, P<.001)$. However, the frequency of contentment ( $r=.103, P=.306$ ) and pleasure $(r=.176, P=.079)$ was not significantly associated with severity of dementia. The apparent curvilinear relationship for these affects (i.e., an increase at the most severe stages of dementia) was not confirmed in regression analyses that included a quadratic term.

\section{Predictors of $Q O L$ in $A D$}

Subjects who had both high positive affect and frequent activity, the high QOL group, comprised $23.1 \%(30 / 125)$ of the sample. Table 3 shows differences between the low and high QOL groups, stratified by living arrangement (nursing home and community). Among the 43 patients in nursing homes, four had high QOL; these patients differed from those with low QOL only in use of antipsychotics $10 \%$ vs $33 \%, P<$ $.001)$. Among the 82 patients in the community, 26 had high QOL. Community patients with high QOL differed from those with low QOL in their superior cognitive and functional status. $55.4 \%$ of the low QOL group needed help or could not perform two of three ADL tasks (dressing, bathing, toileting), compared with only $30.8 \%$ among the high QOL group $(P=.036)$. The low QOL group scored 10 points less, on average, than the high-QOL group on the mMMS ( $P=$ $.011)$. In multivariate logistic regression models, with adjustment for age, education, and gender, functional and cognitive status remained independent predictors of QOL.

\section{QOL and Persistence of Lifelong Activity in Dementia}

As a final exploration of determinants of QOL in $\mathrm{AD}$, we wished to examine the degree to which premorbid features of patient lifestyle might affect QOL. Since years of education is an important indicator of premorbid functioning, we examined the relationship between years of education and activity profiles, controlling for severity of dementia. Patients with $13+$ years of schooling were compared to those with a high school education or less. The two groups differed on the frequency of only one activity, "reading/being read to," as shown in Figure 5. Inspection of Figure 5 shows that patients with high education performed the activity more frequently at every level of dementia severity. The difference between the educational groups, controlling for dementia severity (mMMS) in regression analyses, was significant $(P=.029)$.

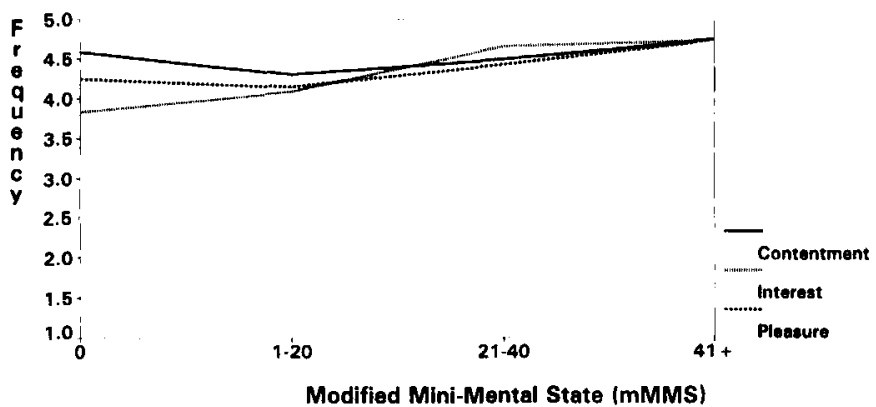

Figure 4. Cognitive deficit in AD: Effect on frequency of positive affect. Mean frequency: 1 , never; $5,>3 \times /$ day.
Table 3. Characteristics of AD Patients, by Residence and QOL Status

\begin{tabular}{llll}
\hline & Low QOL & High QOL & $P$ \\
\hline Nursing Home & & & \\
Sociodemographics & & & \\
$\quad$ Gender (\% female) & 69.2 & 50.0 & .446 \\
$\quad$ Age at intake (yrs) & $75.7( \pm 8.9)$ & $78.0( \pm 5.4)$ & .624 \\
$\quad$ Education (yrs) & $12.7( \pm 3.2)$ & $13.5( \pm 4.9)$ & .656 \\
Features of AD & & & \\
ADL deficit (\%) & 84.6 & 50.0 & .094 \\
mMMS ( $\mu)$ & $16.3( \pm 14)$ & $31.3( \pm 13)$ & .079 \\
$\quad$ EPS (\%) & 53.3 & 33.3 & .555 \\
Antipsychotics (\%) & 33.3 & 0.0 & $<.001$ \\
Community & & & \\
Sociodemographics & & & .088 \\
$\quad$ Gender (\% female) & 62.5 & 42.3 & .509 \\
Age at intake (yrs) & $70.0( \pm 7.8)$ & $71.2( \pm 7.0)$ & .253 \\
Education (yrs) & $13.2( \pm 4.1)$ & $14.1( \pm 3.7)$ & \\
Features of AD & & 30.8 & .036 \\
ADL Deficit (\%) & 55.4 & $30.1( \pm 15)$ & .011 \\
mMMS ( $\mu$ ) & $21.0( \pm 13)$ & .290 \\
EPS (\%) & 35.9 & 22.2 & .673 \\
Antipsychotics $(\%)$ & 15.7 & 12.0 &
\end{tabular}

QOL defined according to (1) above median on frequency of 15 activities and (2) high positive affect ( $>3 \times /$ day on all three positive affects [happy, content, interest ed]). Nursing home patients: 39 low QOL, 4 high QOL; Community-resident patients: 26 low QOI., 56 high QOL.

EPS = presence of clinically significant extrapyramidal signs. ADI deficit = need for help in two of three basic ADLs (dressing, bathing, toileting). mMMS = Modified Mini-Mental Statc exam.

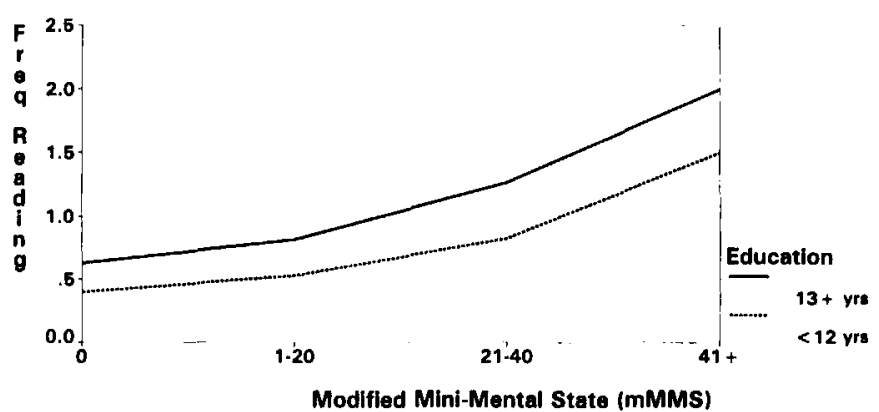

Figure 5. Frequency of reading behavior, by premorbid education and current cognitive status in AD. Mean frequency: 0 , never; $1,<3 \times / \mathrm{wk} ; 2,>3 \times / \mathrm{wk}$.

\section{DISCUSSION}

This research demonstrates that quality of life in demented patients can be elicited reliably from patient proxies and that a measure based on engagement in activity and affective experience captures a great range of variation in the experience of dementia. For a composite measure of high $\mathrm{QOL}$ in $\mathrm{AD}$, we required that patients demonstrate high scores in both an objective and subjective dimension of QOL, that is, activity and positive affect. By this measure, about one-quarter of the patients could be said to have high QOL. Among nursing home patients, high QOL was associated most closely with absence of psychiatric symptoms and nonuse of antipsychotics; among community residents, high QOL was associated with greater function and cognitive ability. In multivariate models, high QOL was associated 
with both functional and cognitive status, but not with patient sociodemographic features.

Dementia clearly affects emotional experience. As dementia severity increased, the frequency of the positive affects (especially interest) declined; however, at the most severe stage of cognitive decline, some caregiver proxies reported an upswing in patient contentment and pleasure. The frequency of negative affects generally increased although anxiety appeared to abate at the lowest levels of cognitive function (with a significant curvilinear regression term for the subset of patients taking antipsychotics). More research on the expression of affects in dementia is clearly warranted.

Activity declined with increasing severity of dementia, but inspection of the individual activity measures showed uneven decline in the frequency of activity. Cognitively demanding activities, such as reading or being read to, significantly declined with increasing severity of dementia. However, activities requiring cueing by caregivers did not show such precipitous declines, and caregiver time with patients increased.

At least one feature of premorbid functioning had an impact on the quality of experience in dementia. This was evident in the relationship between years of schooling and reading behaviors. Patients with post-secondary schooling were more likely to read or be read to at every stage of severity of dementing disease. The relationship between education and reading behaviors retained its significance in multivariate models that controlled for severity of dementia.

These findings should be interpreted in light of the absence of a true gold standard for assessing the validity of the QOL measures. Our information comes from proxies; we relied on their interpretation of participation in an activity or demonstration of an affect. The behaviorally anchored quality of the items used in the research minimizes bias in this regard, but it is important to remember that all our information is refracted through the lens of a caregiver's interpretation of patient behavior. However, confidence in the proxy reports is justified, we feel, because (1) proxies were consistent in their reports of patient behavior (as indicated in the 1-week test-retest reliability study); (2) for patients in institutional care, family and institutional caregiver reports did not differ significantly; (3) the QOL measures were significantly related to disease indicators (i.e., severity of cognitive and functional deficit) and not to sociodemographic indicators. These findings support the reliability and validity of the $\mathrm{QOL}$ measures. Also, Lawton ${ }^{8}$ and Hurley ${ }^{9}$ have provided evidence of the validity of proxy-rated affect using multiple observers in studies of nursing home patients with AD.

A different question asks what the QOL items really measure. When a proxy rates the frequency of an activity, we cannot be sure that a patient engages in the activity in meaningful ways. For example, it is unclear how much a patient said to read truly comprehends or how much a patient attending church or synagogue actually participates in the event. Inquiry about enjoyment helps anchor performance of an event with true participation, but even here there are limits. For example, the low kappa in proxy test-retest reports of patient enjoyment in the case of watching television indicates that it may be difficult to know if an $\mathrm{AD}$ patient truly enjoys certain activities.

The research is relevant to the care of people with AD in a number of ways. First, it clearly establishes variation in the experience of dementia. Patients with similar levels of cogni- tive impairment may have quite different activity patterns and affective experience. These differences may result from modifiable features of disease or environment. For example, caregiver effort is clearly essential for the opportunity for activity, and control of behavioral syndromes clearly offers benefit for patients' affective experience. Second, the research offers a baseline for judging progress and predictors of disease. For example, even patients who have bottomed out on measures of dementia severity (i.e., a score of 0 on the mMMS) show variation in the measures of activity and affect. The QOL measures offer additional endpoints for study of disease course.

In short, this research shows the value of a QOL inquiry among demented patients. Even with this limited inquiry, we feel that many assumptions about the experience of dementia need to be re-evaluated. Patients with dementia show great variability in activity and affect and, hence, in QOL. This variability is determined by severity of disease, but also by caregiver effort, the different demands of activities, premorbid activity levels, and the presence of behavioral syndromes associated with $\mathrm{AD}$. These issues will become clearer as longitudinal data become available.

\section{ACKNOWLEDGMENTS}

Research was supported by federal grants AGO7370 and RR00645, the Charles S. Robertson Gift for Alzheimer's Disease from the Banbury Fund, and the Alzheimer's Association (FSA-93-026).

\section{REFERENCES}

1. Binstock RH, Post SG, Whitehouse PJ. Dementia and Aging: Ethics, Values, Policy Choices. Baltimore: Johns Hopkins Press, 1992.

2. Buchanan AE, Brock DW. Deciding for Others. The Ethics of Surrogate Decision-making. Cambridge: Cambridge University Press, 1989.

3. Dresser R, Whitehouse PJ. The incompetent patient on the slippery slope. Hastings Cent Rep 1994;24:6-12.

4. Blessed G, Tomlinson BE, Roth M. The association between quantitative measures of dementia and of senile changes in the cerebral grey matter of elderly subjects. Br J Psychiatry 1968;225:797-811.

5. Folstein MF, Folstein SE, McHugh PR. Mini-Mental State: A practical method for grading the cognitive state of patients for the clinician. J Psychiatr Res $1975 ; 12: 189-198$.

6. Stern Y, Sano M, Paulson J, Mayeux R. Modified Mini-Mental State Examination: Validity and reliability. Neurology 1987;37(suppl):179.

7. Teri L, Logsdon RG. Identifying pleasant activities for Alzheimer's disease patients: The Pleasant Events Schedule-AD. Gerontologist 1991;31:124-127.

8. Lawton MP. Quality of life in Alzheimer's disease. Alzheimer Dis Assoc Disord 1994;8(suppl):S138-150.

9. Hurley AC, Volicer BJ, Hanarahan PA et al. The assessment of discomfort in advanced Alzheimer's patients. Res Nurs Health 1992;15:369-377.

10. Erickson P, Wilson RW, Seitz F et al. Years of healthy life: A measure of healthy life span for Health People 2000. In: Proc 1993 Public Health Conference on Records and Statistics. Bethesda, MD: Centers for Disease Control and Prevention, 1993, pp 21-27.

11. Hamilton M. A rating scale for depression. J Neurol Neurosurg Psychiatry $1960 ; 23: 56-62$.

12. Devanand DP, Miller L, Richards $M$ et al. The Columbia University scale for psychopathology in Alzheimer's disease. Arch Neurol 1992;49:371-376.

13. Hughes CP, Berg I, Danziger WL et al. A new clinical scale for the staging of dementia. Br J Psychol 1982;140:566-572.

14. Stern $Y$, Folstein $M$, Albert $M$ et al. Multi-center study of predictors of disease course in Alzheimer's disease (the "Predictor's Study"): I. Study design, cohort description, and intersite comparisons. Alzheimer Dis Rel Disord $1993 ; 7: 3-21$.

15. Richards $M$, Folstein $M$, Albert $M$ et al. Multi-center study of predictors of discase course in Alzheimer's discase (the "Predictor's Study"): II. Baseline findings. Alzhcimer Dis Rel Disord 1993;7:22-32.

16. Fleiss JL. Statistical Methods for Rates and Proportions. New York: John Wiley \& Sons, 1981. 